\title{
A Bivariate Maintenance Policy for Multi-state Repairable Systems with Monotone Process
}

\author{
Mimi Zhang ${ }^{1 *}$, Min Xie ${ }^{1}$, Fellow, IEEE, and Olivier Gaudoin ${ }^{2}$ \\ [mmzhang5@student.cityu.edu.hk, minxie@cityu.edu.hk, Olivier.Gaudoin@imag.fr] \\ ${ }^{1}$ Department of Systems Engineering and Engineering Management, City University of Hong \\ Kong, Hong Kong, China \\ ${ }^{2}$ Grenoble University, Laboratoire Jean Kuntzmann, BP 53-38 041 Grenoble Cedex 9, France
}

\begin{abstract}
In this paper, a sequential failure limit maintenance policy for a repairable system is studied. The system is assumed to have $k+1$ states, including one working state and $k$ failure states, and the multiple failure states are classified by, e.g., failure severity or failure cause. The system will be replaced at the $N$ th failure and corrective maintenance is conducted immediately at each of the first $N-1$ failures. A reliability-centered preventive maintenance schedule is proposed in which, between two adjacent failures, a preventive maintenance action is taken as soon as the system reliability drops to a critical reliability $R$. Both preventive maintenance and corrective maintenance are assumed to be imperfect. Increasing and decreasing geometric processes are introduced to characterize the efficiency of these two types of maintenance. The objective is to derive an optimal maintenance policy $(R, N)^{*}$ such that the long-run expected cost per unit time

\footnotetext{
* Corresponding author.
}

Tel: $+852-5446-5352$.
\end{abstract}


is minimized. The explicit expression of the average cost rate is derived, and the corresponding optimal maintenance policy can be determined analytically or numerically. A numerical example is given to illustrate the theoretical results and the procedure. The decision model shows its adaptability to different possible characteristics of the maintained system.

Key words: Geometric process; Quasi-renewal process; Multiple failure states; Sequential failure limit policy.

\section{ACRONYMS \& NOTATION}

$V_{n} \quad$ the number of preventive maintenance in the $n$th repair cycle

$x_{n}^{j} \quad$ the working time between the $(j-1)$ th and the $j$ th preventive maintenance in the $n$th repair cycle

$X_{n} \quad$ the working time between the $V_{n}$ th preventive maintenance and the corrective maintenance in the $n$th repair cycle

$Y_{n} \quad$ the length of the corrective maintenance in the $n$th repair cycle

$S_{n} \quad$ the failure type in the $n$th repair cycle

$C_{p} \quad$ cost of each preventive maintenance

$c_{f} \quad$ corrective maintenance cost rate due to the down-time

$c_{i} \quad$ failure damage of failure type $i, i=1, \ldots, k$

C replacement cost

a the impact of the preventive maintenance on system's lifetime distribution

b the impact of the preventive maintenance on the duration of corrective maintenance

$a_{i} \quad$ the impact on the system's lifetime distribution by each occurrence of failure type $i$, 
$i=1, \ldots, k$

$b_{i} \quad$ the impact on the duration of corrective maintenance by each occurrence of failure type $i, i=1, \ldots, k$

$p_{i} \quad$ the probability of occurrence of the $i$ th type failure, $i=1, \ldots, k$

$L_{n} \quad$ the time to failure in the $n$th repair cycle

PM preventive maintenance

CM corrective maintenance

GP geometric process

QRP quasi-renewal process

\section{Introduction}

For a repairable deteriorating system, the most advanced maintenance strategies rely on the monitoring of a measurable degradation process of the system and base the maintenance decisions on the level of degradation. Generally, such a condition-based maintenance policy is more efficient than a maintenance policy based on the system age and on the knowledge of the underlying lifetime distribution. However, building a degradation database is very expensive and some legacy systems still lack the capability of acquiring sensor-based information. Moreover, it is unrealistic to establish a condition-based maintenance program for each small but critical element such as the horizon of an airplane and the radar altimeter of a satellite. This is, however, not the case with failure time data which can be easily retrieved from historical maintenance records (Gebraeel et al. [1]). In view of this, we consider a maintenance strategy which is applicable to those systems inaccessible to the condition-based maintenance program. Note that 
the preliminarily used lifetime distributions can be updated by the subsequent real-time sensory signals.

It is well-known that the maintenance of a deteriorating system is usually not perfect, restoring the system's operation state to somewhere between as good as new and as bad as old (see [2]-[4]). For a repairable system, a common phenomenon is that the successive operating times of the system after repair become shorter and shorter, whereas the mean lengths of the successive repair times are increasing. To model the maintenance efficiency with this characteristic, Lam [5] introduces a geometric process (GP) repair model. It should be noted that Wang and Pham [6] propose a quasi-renewal process (QRP) to modulate imperfect maintenance process. Conceptually different as they are, the GP and the QRP share the same theoretical basis. As Lam put this idea out first, we use the notation "geometric process" in the following. The GP and the QRP models have been studied by many researchers. The readers are referred to [7]-[10] for more details.

The GP and the QRP were originally introduced to characterize the effectiveness of corrective maintenance $(\mathrm{CM})$. Modulating imperfect preventive maintenance (PM) processes via the GP, however, has received surprisingly little attention until very recently. Wang and Pham [11] modulate the imperfect PM by a quasi-renewal process in which the degradation critical threshold is raised proportionally after each PM. Doyen and Gaudoin [12] also pointed out that the quasi-renewal process can be generalized to imperfect PM by introducing a positive parameter characterizing the PM efficiency. Yet, this article just presents a general framework and does not give much detailed discussions on this generalized model. In this current paper, we propose a maintenance strategy, including both imperfect CM and imperfect PM, and assess the efficiency of these two maintenance actions both in the context of GP. As the PM and the CM 
are only different in the sense that the PM is planned while the CM is unplanned, it is reasonable to characterize PM and CM efficiency both using the GP, with different impact factors.

In recent years, there has been growing research interest on multi-state reliability theories, models and optimization algorithms. Derman [13] is among the first to investigate a multi-state model, where the deterioration of the system was described as the movement from state to state by a Markov chain. More works on multi-state systems can be found in [14]-[17]. A special type of multi-state system model, which we will deliberate in this paper, is defined as follows: A degenerative system has $k+1$ states with one working state and $k$ different failure states (Zhang and Wang [18]). The occurrences of these $k$ failure states are stochastic and mutually exclusive. For example, in the context of gracefully degrading systems, a failure may be classified by its severity and thus the cost related to each failure type is different. Another example is that a failure can be classified by its cause and thus the treatment related to each failure type is different. Lam et al. [19] studied a monotone process repair model for such system with $k$ failure modes. They applied a replacement policy based on the number of failures of the system in which the system state after repair cannot be "as good as new". They showed that the repair model for the multi-state degenerative system forms a general monotone process repair model which includes the GP repair model as a special case. Several extensions of this multi-state model have been investigated in Zhang et al. [20] and Zhang and Wang [18]. However, the multi-state failure system subjected to imperfect CM and imperfect PM, both in the form of GP, is a blank topic of interest.

Conventional PM is scheduled periodically and it often holds the same time interval $T$ for PM actions (Sheu et al. [21]). However, because the PM is generally imperfect and it cannot restore the system to as good as new, the age-dependent or periodic PM policy is unavoidably 
ineffective. On the other hand, as Wang [22] pointed out, the optimal maintenance policy must be based not only on cost rate but also on reliability measures. Sometimes when the maintenance cost rate is minimized the system reliability measures are so low that they are not acceptable in practice. This leads to the sequential failure limit PM policy presented herein. Under sequential failure limit PM policy, the PM actions are scheduled so that the system does not drop below a critical reliability. Many literature works focus on reliability centered PM policy. Zhou et al. [23] integrates sequential imperfect maintenance policy into condition based predictive maintenance policy and an imperfect PM is performed whenever the system reliability reaches the threshold $R$. In Liao et al. [24], it is assumed that the system's reliability could be monitored continuously and the imperfect PM is performed whenever the system's reliability reaches the threshold $R$. Doyen and Gaudoin [12] also mentioned a sequential failure limit PM policy in which the system is preventively maintained as soon as a reliability indicator exceeds a predetermined threshold.

The maintenance strategy in this present paper is a combination of failure limit policy and repair number counting policy (Wang [22]). The system is replaced by a new one at the $N$ th failure and $\mathrm{CM}$ is conducted at the first $(N-1)$ failures. PM is performed whenever the system reliability drops to a critical threshold $R$. Both the CM and the PM are assumed to be imperfect. That is, the successive working times of the system after repair will become shorter and shorter. We further assume that the consecutive corrective repair times of the system become longer and longer. Because PM actions are pre-scheduled, the PM times are assumed to be negligible. One interpretation of replacing at the $N$ th failure is that when a new system is put into operation, the first $N$ repairs at failures will be performed at a low cost, because the system is young. It will be in a good operating state and should not need any major repairs for some period. After $N$ repairs, this system will be in a bad condition and then it is more economical to replace it by a new one. 
The policy decision variables are $R$ and $N$. The purpose is to determine the optimal policy parameters $(R, N)^{*}$ such that the long-run expected cost per unit time is minimized.

This paper is organized as follows. In Section 2, a monotone process model for a deteriorating system, which has one working state and $k$ failure states, is introduced. In Section 3 , an explicit expression of the average cost rate is derived. The optimal policy $(R, N)^{*}$ can be determined numerically or analytically for minimizing the average cost. Section 4 illustrates the maintenance policy via an example. The last section concludes the paper.

\section{Model assumptions}

For easy reference, we first state the definitions of stochastic order and GP as follows (see Ross [25]):

Definition 1. Given two random variables $X$ and $Y, X$ is said to be stochastically larger than $Y$ if

$$
P(X>t) \geq P(Y>t), \quad \forall t \in R,
$$

and it is denoted by $X \geq_{s t} Y$ or $Y \leq_{s t} X$. We say that a stochastic process $\left\{X_{n}, n=1,2, \ldots\right\}$ is stochastically decreasing if $X_{n} \geq_{s t} X_{n+1}$ and stochastically increasing if $X_{n} \leq_{s t} X_{n+1}$, for all $n=1,2, \ldots$

Definition 2. Assume that $\left\{X_{n}, n=1,2, \ldots\right\}$ is a sequence of independent non-negative random variables. If the distribution function of $X_{n}$ is $F_{n}(t)=F\left(a^{n-1} t\right)$ for some $a>0$ and all $n=$ $1,2, \ldots$, then $\left\{X_{n}, n=1,2, \ldots\right\}$ is called a geometric process. Furthermore, if $a>1,\left\{X_{n}, n=\right.$ $1,2, \ldots\}$ is called stochastically decreasing, i.e. 


$$
X_{n} \geq_{s t} X_{n+1} \text {, for all } n=1,2, \ldots
$$

If $0<a<1,\left\{X_{n}, n=1,2, \ldots\right\}$ is called stochastically increasing, i.e.

$$
X_{n} \leq_{s t} X_{n+1}, \text { for all } n=1,2, \ldots
$$

If $a=1$, the geometric process becomes a renewal process.

Our model assumptions are listed below.

Assumption 1. At the beginning, a new system is installed and will be replaced at the $N$ th failure by a new one with negligible replacement time.

The time interval between the $(m-1)$ th replacement and the $m$ th replacement of the system is called the $m$ th renewal cycle, $m=1,2, \ldots$ Let $T_{1}$ be the first replacement time point of the system. Let $T_{m}(m \geq 2)$ be the time duration between the $(m-1)$ th replacement and the $m$ th replacement of the system. Obviously, $\left\{T_{1}, T_{2}, \ldots\right\}$ forms a renewal process.

Assumption 2. The system is a multi-state system with one working state and $k$ failure states. We use $0,1,2, \ldots, k$ to denote the working state, the first-type failure state, the second-type failure state, ..., and the $k$ th-type failure state. If the system fails, then with probability $p_{i}$ the system will be in state $i, i=1, \ldots, k$, and $\sum_{i=1}^{k} p_{i}=1$.

As we can always arrange the $k$ failure states in order of failure severity we might assume that failure type $i+1$ is more serious than failure type $i$ for $i=1,2, \ldots, k-1$. Thus, state 1 is a failure state with the lowest severity.

Assumption 3. The PM is executed as soon as the system reliability drops to the critical threshold $R$. The CM commences immediately at the system's failure. The PM is assumed to 
take negligible time. Both PM and CM are assumed to be imperfect. Each PM is assumed to have an effect $a$ on working time distribution and an effect $b$ on repair time distribution. Each $\mathrm{CM}$ is assumed to have an effect $a_{s}$ on working time distribution and an effect $b_{s}$ on repair time distribution, where $s$ indicates the failure type.

The time interval between the completion of the $(n-1)$ th corrective maintenance and the completion of the $n$th corrective maintenance is called the $n$th repair cycle of the system, $n=1,2, \ldots N$. Let random variable $V_{n}$ denote the number of PM conducted in the $n$th repair cycle. Given $V_{n}=v_{n}$, let $x_{n}^{j}, X_{n}$ and $Y_{n}$ be, respectively, the working time before $j$ th PM, the working time before $n$th $\mathrm{CM}$ and the length of the $n$th $\mathrm{CM}\left(j=1, \ldots, v_{n}\right)$. A possible course of the $n$th repair cycle is shown in Fig. 1.

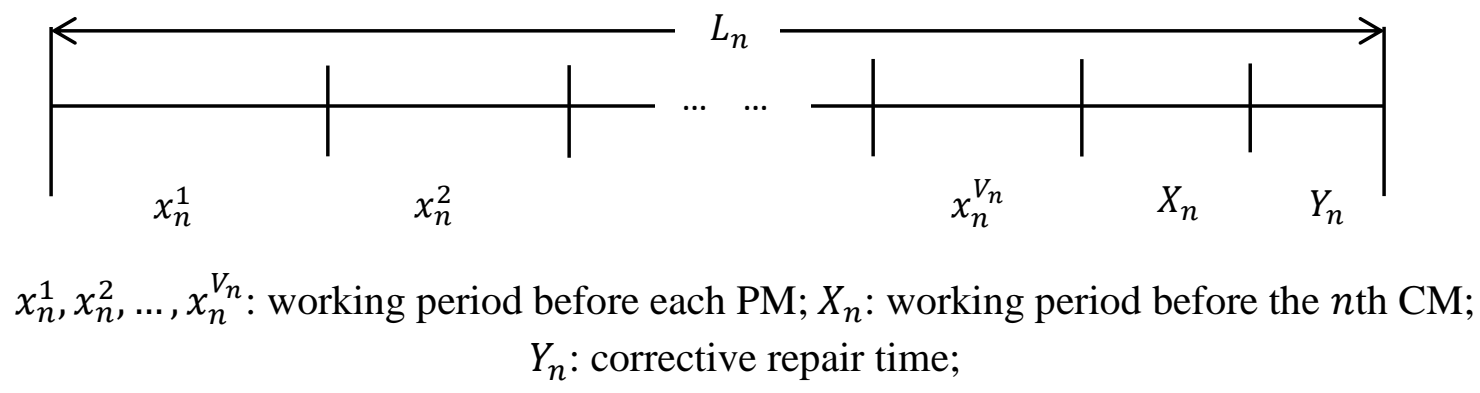

Fig. 1. A possible course of the system in $n$th repair cycle.

Assumption 4. $\left\{X_{1}, X_{2}, \ldots, X_{k} \ldots\right\}$ and $\left\{Y_{1}, Y_{2}, \ldots, Y_{k} \ldots\right\}$ are two independent processes.

Assumption 5. Given that the system's working time after the $j$ th PM in the $n$th repair cycle follows distribution $H(t), t \geq 0$, and that the system survives beyond the PM threshold, we have $x_{n}^{j+1}=H^{-1}(1-R)$ and that the system's working time after the $(j+1)$ th PM follows 
distribution $H(a t), a \geq 1$. Parameter $a$ implies that as the system deteriorates the working time will be shorter and shorter. Given $X_{n} \sim H(t)(t \geq 0)$, with failure type $s_{n}$, the system's working time at the beginning of the $(n+1)$ th repair cycle follows distribution $H\left(a_{s_{n}} t\right)$, where $s_{n} \in$ $\{1,2, \ldots, k\}$ and $1 \leq a \leq a_{1} \leq a_{2} \leq \cdots \leq a_{k}$. Parameter $a_{i}$ represents the impact on the system's lifetime distribution by each occurrence of failure type $i$.

Suppose that the working time of the system at the beginning of a renewal cycle has distribution function $F(t)$. In the $n$th repair cycle, we have

$$
\begin{gathered}
x_{n}^{j}=\frac{F^{-1}(1-R)}{a^{\sum_{i=1}^{n-1} v_{i}+j-1} \prod_{i=1}^{n-1} a_{s_{i}}} \\
P\left(X_{n} \leq t \mid V_{1}=v_{1}, \ldots, V_{n}=v_{n}, S_{1}=s_{1}, \ldots, S_{n-1}=s_{n-1}\right)=\frac{F\left(a^{\sum_{i=1}^{n} v_{i}} \prod_{i=1}^{n-1} a_{s_{i}} t\right)}{1-R},
\end{gathered}
$$

where $j=1, \ldots, v_{n}, 0<t<\tau_{n}, s_{1}, s_{2}, \ldots, s_{n-1} \in\{1,2, \ldots, k\}$ and

$$
\tau_{n}=\frac{F^{-1}(1-R)}{a^{\sum_{i=1}^{n} v_{i}} \prod_{i=1}^{n-1} a_{s_{i}}}
$$

Assumption 6. Given $Y_{n-1} \sim H(t)(t>0), V_{n}=v_{n}$ and failure type $s_{n}$, the CM time $Y_{n}$ follows distribution $H\left(b^{v_{n}} b_{s_{n}} t\right)$, where $s_{n} \in\{1,2, \ldots, k\}$ and $0<b_{k} \leq \cdots \leq b_{1} \leq b \leq 1$. Parameter $b$ implies that as the system deteriorates, the repair time will be longer and longer. Parameter $b_{i}$ represents the impact on the repair time by each occurrence of failure type $i$.

Similarly, suppose that the CM duration of a new system has cumulative distribution function $G(t)$, namely $G(t)$ is a generic cumulative distribution function of the repair times. In the $n$th repair cycle, we have 


$$
P\left(Y_{n} \leq t \mid V_{1}=v_{1}, \ldots, V_{n}=v_{n}, S_{1}=s_{1}, \ldots, S_{n}=s_{n}\right)=G\left(b^{\sum_{i=1}^{n} v_{i}} \prod_{i=1}^{n} b_{s_{i}} t\right)
$$

where $t>0$ and $s_{1}, s_{2}, \ldots, s_{n} \in\{1,2, \ldots, k\}$.

Denote the cost of each occurrence of failure by $C_{n}(n=1,2, \ldots)$. We assume that $C_{n}$ are independent and identically distributed random variables with distribution $P\left(C_{n}=c_{i}\right)=p_{i}$, $i=$ $1, \ldots, k$. The corrective maintenance cost rate, due to down-time, is denoted by $c_{f}$. The replacement cost in each renewal cycle is $C$, and the cost for each PM is $C_{p}$.

The following theorem shows that $\left\{X_{1}, X_{2}, \ldots, X_{n} \ldots\right\}$ is a monotone decreasing process and $\left\{Y_{1}, Y_{2}, \ldots, Y_{n} \ldots\right\}$ is a monotone increasing process.

Theorem 1. For $n=1,2 \ldots$, and $\forall t>0$, we have

$$
\begin{aligned}
& P\left(X_{n}>t\right) \geq P\left(X_{n+1}>t\right), \\
& P\left(Y_{n}>t\right) \leq P\left(Y_{n+1}>t\right) .
\end{aligned}
$$

The proof of this theorem is given in Appendix A.

\section{Average cost rate under policy $(R, N)$}

In this section, we consider the bivariate policy $(R, N)$ based on the critical threshold $R$ and the failure number $N$. Our objective is to determine the optimal policy $(R, N)^{*}$ such that the long-run expected cost per unit time is minimized. 
Let $C(R, N)$ denote the average cost rate under policy $(R, N)$. According to the renewal reward theorem (see Ross [26]), we have

$$
C(R, N)=\frac{\text { expected cost incurred in a renewal cycle }}{\text { expected lenght of the remewal cycle }}=\frac{E U}{E W}
$$

where $U$ and $W$ denote the total cost in a renewal cycle and the length of a renewal cycle, respectively.

According to the assumptions, we have

$$
W=\left[\sum_{i=1}^{N} \sum_{j=1}^{V_{i}} x_{i}^{j}+\sum_{i=1}^{N} X_{i}\right]+\sum_{i=1}^{N-1} Y_{i}
$$

The first part at the right side of the equation denotes the total working time while the second part denotes the total repair time of a renewal cycle. The total cost of a renewal cycle is given by

$$
U=C+\sum_{i=1}^{N} \sum_{j=1}^{V_{i}} C_{p}+c_{f} \sum_{i=1}^{N-1} Y_{i}+\sum_{i=1}^{N} C_{i}
$$

Thus, the average cost rate $C(R, N)$ can be written as

$$
C(R, N)=\frac{C+C_{p} \sum_{i=1}^{N} E V_{i}+c_{f} \sum_{i=1}^{N-1} E Y_{i}+\sum_{i=1}^{N} E C_{i}}{\sum_{i=1}^{N} E\left(\sum_{j=1}^{V_{i}} x_{i}^{j}\right)+\sum_{i=1}^{N} E X_{i}+\sum_{i=1}^{N-1} E Y_{i}}
$$

All the expectations in function $C(R, N)$ are stated below and their proofs are given in Appendix B.

$$
E\left(\sum_{i=1}^{N} C_{i}\right)=N \sum_{i=1}^{k} c_{i} p_{i}
$$




$$
\begin{gathered}
E V_{n}=\frac{R}{1-R} ; \\
E\left(X_{n}\right)=\left(\frac{p_{1}}{a_{1}}+\cdots+\frac{p_{k}}{a_{k}}\right)^{n-1}(1-R)^{n-1} \frac{\lambda(R) a^{n}}{(a-R)^{n}}, \quad \lambda(R)=\int_{0}^{F^{-1}(1-R)} t d F(t), \quad a>R ; \\
E\left(Y_{n}\right)=(1-R)^{n} \frac{u b^{n}}{(b-R)^{n}}\left(\frac{p_{1}}{b_{1}}+\cdots+\frac{p_{k}}{b_{k}}\right)^{n}, \quad u=\int_{0}^{+\infty} t d G(t), \quad b>R ; \\
E\left(x_{n}^{j}\right)=\left(\frac{p_{1}}{a_{1}}+\cdots+\frac{p_{k}}{a_{k}}\right)^{n-1}(1-R)^{n-1}\left(\frac{a}{a-R}\right)^{n-1} \frac{R^{j} \times F^{-1}(1-R)}{a^{j-1}} \quad j=1, \ldots, v_{n}, \quad a>R .
\end{gathered}
$$

For simplicity of reference, we define $A$ and $B$ as follows:

$$
A=\frac{p_{1}}{a_{1}}+\cdots+\frac{p_{k}}{a_{k}} \quad B=\frac{p_{1}}{b_{1}}+\cdots+\frac{p_{k}}{b_{k}}
$$

Theorem 2. Under policy $(R, N)$, the explicit expression of $C(R, N)$ is

$$
C(R, N)=\frac{C+N C_{p} R /(1-R)+c_{f} \Psi_{3}+N \sum_{i=1}^{k} c_{i} p_{i}}{\Psi_{1}+\Psi_{2}+\Psi_{3}},
$$

where

$$
\begin{gathered}
\Psi_{1}=\sum_{i=1}^{N} E\left(\sum_{j=1}^{V_{i}} x_{i}^{j}\right)=\frac{F^{-1}(1-R) \times a R}{a-R} \times \frac{1-\left[A(1-R) \frac{a}{a-R}\right]^{N}}{1-A(1-R) \frac{a}{a-R}}, \\
\Psi_{2}=\sum_{i=1}^{N} E X_{i}=\frac{\lambda(R) a}{a-R} \times \frac{1-\left[A(1-R) \frac{a}{a-R}\right]^{N}}{1-A(1-R) \frac{a}{a-R}} \\
\Psi_{3}=\sum_{i=1}^{N-1} E Y_{i}=B(1-R) \frac{u b}{b-R} \times \frac{1-\left[B(1-R) \frac{b}{b-R}\right]^{N-1}}{1-B(1-R) \frac{b}{b-R}} .
\end{gathered}
$$


For a proof, see Appendix C.

From the expression of the cost rate function $C(R, N)$ we can see that the form of the generic distribution function of the $\mathrm{CM}$ duration, $G(\cdot)$, makes no difference as long as the mean $u$ remains unchanged.

Remark 1. We can solve the bivariate optimization problem $C(R, N)$ via two univariate optimization procedures. When $N$ is fixed, e.g., $N=n, C(R, N)$ can be written as a function of $R$

$$
C(R, N)=C_{n}(R), \quad n=1,2,3, \ldots
$$

and the optimal reliability is thus a function of $n, R_{n}^{*}$. Therefore, for a fixed $n$, we can find $R_{n}^{*}$ by analytical or numerical methods such that $C_{n}\left(R_{n}^{*}\right)$ is minimized. By choosing $N$ to be $1,2, \ldots, n, \ldots$, we can find $R_{1}^{*}, R_{2}^{*}, \ldots, R_{n}^{*}, \ldots$ respectively such that the corresponding $C_{1}\left(R_{1}^{*}\right), C_{2}\left(R_{2}^{*}\right), \ldots, C_{n}\left(R_{n}^{*}\right), \ldots$ are minimized. Because of the degeneration, the total length of a renewal cycle is limited (see Wang and Pham [7]). Therefore, we can determine the minimum of the long-run expected cost rate by comparing the values of $C_{1}\left(R_{1}^{*}\right), C_{2}\left(R_{2}^{*}\right), \ldots, C_{n}\left(R_{n}^{*}\right), \ldots$.

Remark 2. Given the deteriorating trajectories $\left(V_{1}=v_{1}, \ldots, V_{n-1}=v_{n-1}, S_{1}=s_{1}, \ldots, S_{n-1}=\right.$ $\left.s_{n-1}\right)$, it is of interest to derive the survival function $P\left(L_{n}>t\right) \equiv P\left(L_{n}>t \mid V_{1}=v_{1}, \ldots, V_{n-1}=\right.$ $\left.v_{n-1}, S_{1}=s_{1}, \ldots, S_{n-1}=s_{n-1}\right)$, where $L_{n}=\sum_{j=1}^{V_{n}} x_{n}^{j}+X_{n}$ denotes the time to failure of the $n$th repair cycle. We have

$$
x_{n}^{j}=\frac{F^{-1}(1-R)}{a^{\sum_{i=1}^{n-1} v_{i}+j-1} \prod_{i=1}^{n-1} a_{s_{i}}}, \quad j=1,2, \cdots, n, \cdots .
$$

For $0 \leq t<x_{n}^{1}$, 


$$
P\left(L_{n}>t\right)=1-F\left(a^{\sum_{i=1}^{n-1} v_{i}} \prod_{i=1}^{n-1} a_{s_{i}} t\right) .
$$

For $\sum_{i=1}^{j-1} x_{n}^{i} \leq t<\sum_{i=1}^{j} x_{n}^{i}, j=2,3, \ldots$,

$$
\begin{aligned}
P\left(L_{n}>t\right)= & \sum_{v_{n}=0}^{+\infty} P\left(L_{n}>t \mid V_{n}=v_{n}\right) \times R^{v_{n}}(1-R) \\
& =\sum_{v_{n}=j-1}^{+\infty} P\left(L_{n}>t \mid V_{n}=v_{n}\right) \times R^{v_{n}}(1-R) \\
& =\left[1-F\left(a^{\sum_{i=1}^{n-1} v_{i}+j-1} \prod_{i=1}^{n-1} a_{s_{i}} t\right)\right] \times R^{j-1}(1-R)+\sum_{v_{n}=j}^{+\infty} R^{v_{n}}(1-R) \\
& =\left[1-F\left(a^{\sum_{i=1}^{n-1} v_{i}+j-1} \prod_{i=1}^{n-1} a_{s_{i}} t\right)\right] \times R^{j-1}(1-R)+R^{j} .
\end{aligned}
$$

Another interesting measurement of system reliability is the mean time to failure (MTTF), which is easy to give

$$
\begin{aligned}
& E\left[\sum_{j=1}^{V_{n}} x_{n}^{j}+X_{n} \mid V_{1}=v_{1}, \ldots, V_{n-1}=v_{n-1}, S_{1}=s_{1}, \ldots, S_{n-1}=s_{n-1}\right] \\
& =E_{V_{n}}\left[E\left[\sum_{j=1}^{V_{n}} x_{n}^{j}+X_{n} \mid V_{1}=v_{1}, \ldots, V_{n-1}=v_{n-1}, V_{n}=v_{n}, S_{1}=s_{1}, \ldots, S_{n-1}=s_{n-1}\right]\right] \\
& =E_{V_{n}}\left[\frac{\lambda(R) /(1-R)}{a^{\sum_{i=1}^{n} v_{i}} \prod_{i=1}^{n-1} a_{s_{i}}}+\sum_{j=1}^{V_{n}} \frac{F^{-1}(1-R)}{a^{\sum_{i=1}^{n-1} v_{i}+j-1} \prod_{i=1}^{n-1} a_{s_{i}}}\right] \\
& =\frac{\lambda(R) / R+F^{-1}(1-R)}{a^{\sum_{i=1}^{n-1} v_{i}} \prod_{i=1}^{n-1} a_{s_{i}}} \times \frac{a R}{a-R} .
\end{aligned}
$$




\section{Numerical Example}

To demonstrate the model and the methodology developed in this paper, a numerical example is discussed in this section. In the following analysis we assume that the original survival time distribution is known, and its parameters are given (estimated). For illustrative purpose, we consider a deteriorating repairable system with only three states, namely two failure states and one working state $(k=2)$.

Assume that at the beginning of a renewal cycle, the working time of a new system has a Weibull distribution

$$
F(t)=1-\exp \left(-\left(\frac{t}{\beta}\right)^{\alpha}\right)
$$

with parameters $\alpha=1.5$ and $\beta=2000$. Assuming that $p_{1}=0.45, p_{2}=0.55, a=1.03$, $a_{1}=1.1, a_{2}=1.2, b=0.98, b_{1}=0.9, b_{2}=0.8$, we have

$$
A=\frac{p_{1}}{a_{1}}+\frac{p_{2}}{a_{2}}=0.7992 \quad B=\frac{p_{1}}{b_{1}}+\frac{p_{2}}{b_{2}}=1.3125 .
$$

We assume that the other parameters are obtained as $C_{p}=5000, c_{f}=100, c_{1} p_{1}+c_{2} p_{2}=$ $10000, C=500000, u=240$.

For each value of $N$, we calculate an optimal $R_{N}^{*}$ which minimizes the expected long-run cost rate. Fig. 2 shows the result. The $x$-axis denotes the number of failures (repair cycles) and the $y$-axis denotes the corresponding minimum cost rate. It is obvious that $(R, N)^{*}=(0.6488,6)$ is a global optimum at which $C(R, N)$ is minimized. $C(R, N)^{*}=78.3066$ is the minimum of the 
expected long-run cost rate (see Table 1). The maintenance policy can thus be interpreted as: the system will be replaced by a new one upon its sixth failure and CM is conducted immediately at the first five failures. In order to avoid failures and to reduce cost, the system will be preventively maintained once its reliability drops to 0.6488 .

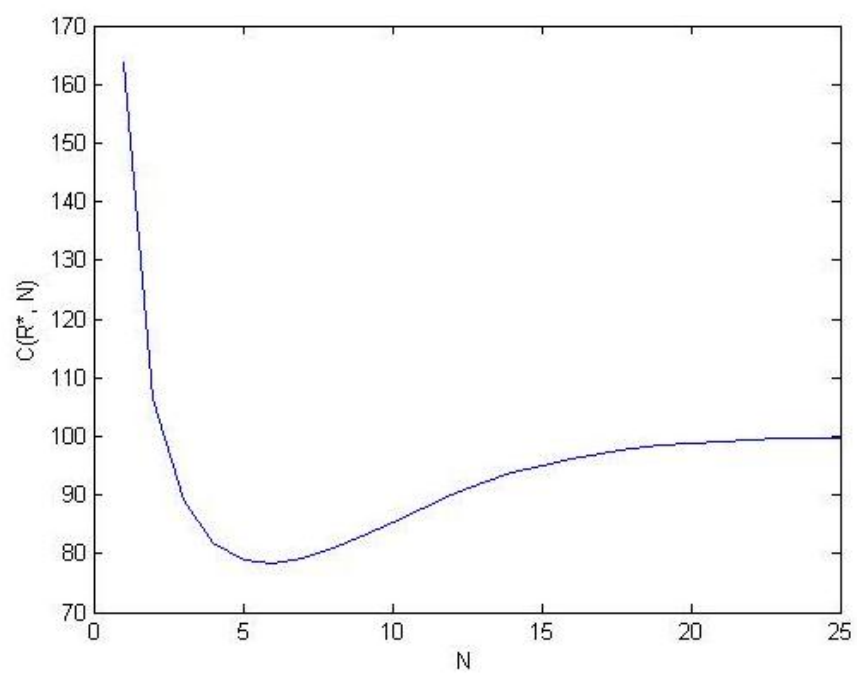

Fig. 2. The plot of cost rate against $\boldsymbol{N}$.

Table 1. The optimal reliability and the corresponding minimized expected long-run cost rate for

\begin{tabular}{ccccccccc}
\hline \multicolumn{10}{c}{ each value of $\boldsymbol{N}$. } \\
\hline$N$ & 1 & 2 & 3 & 4 & 5 & 6 & 7 & 8 \\
$R^{*}$ & 0.91 & 0.85 & 0.79 & 0.74 & 0.69 & 0.65 & 0.61 & 0.57 \\
$C\left(R^{*}, N\right)$ & 163.57 & 106.53 & 89.01 & 81.75 & 78.86 & 78.31 & 79.15 & 80.84 \\
\hline$N$ & 9 & 10 & 11 & 12 & 13 & 14 & 15 & 16 \\
$R^{*}$ & 0.52 & 0.49 & 0.46 & 0.43 & 0.40 & 0.37 & 0.34 & 0.32 \\
$C\left(R^{*}, N\right)$ & 83.01 & 85.38 & 87.76 & 89.99 & 91.98 & 93.69 & 95.11 & 96.27 \\
\hline$N$ & 17 & 18 & 19 & 20 & 21 & 22 & 23 & 24 \\
$R^{*}$ & 0.30 & 0.28 & 0.27 & 0.25 & 0.24 & 0.23 & 0.26 & 0.21 \\
$C\left(R^{*}, N\right)$ & 97.18 & 97.89 & 98.43 & 98.84 & 99.15 & 99.38 & 99.55 & 99.67 \\
\hline
\end{tabular}


Fig. 3 shows the variety of the average cost rate based on bivariate policy $(R, N)$. It can be seen that the optimal policy can be found uniquely from the cost rate surface.

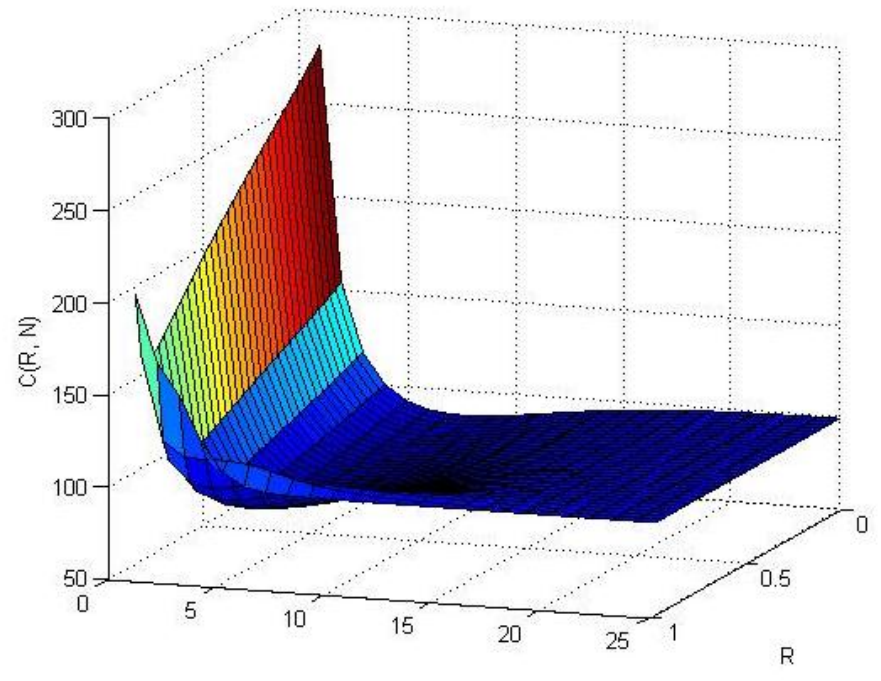

Fig. 3. The plot of cost rate versus $\boldsymbol{R}$ and $\boldsymbol{N}$.

Fig. 4 demonstrates the decreasing property of process $\left\{X_{1}, X_{2}, \ldots, X_{n} \ldots\right\}$. Under policy $(R, N)^{*}=(0.6488,6)$, we first randomly generate six failure types and then simulate the working process $\left\{x_{1}^{1}, x_{1}^{2}, \ldots, x_{1}^{v_{1}}, X_{1}, x_{2}^{1}, x_{2}^{2}, \ldots, x_{2}^{v_{2}}, X_{2}, \ldots, x_{6}^{1}, x_{6}^{2}, \ldots, x_{6}^{v_{6}}, X_{6}\right\}$. Define cumulative working time $T_{n}$ as $T_{n}=\sum_{i=1}^{n} X_{i}, n=1,2, \ldots, 6$. Fig. 4 depicts the cumulative working time $T_{n}$ versus the number of repair cycles $n$, in which there are 20 renewal cycles. The shape of the curves shows the decreasing property of process $\left\{X_{1}, X_{2}, \ldots, X_{n} \ldots\right\}$. More detailed results can be found in Table 2. 


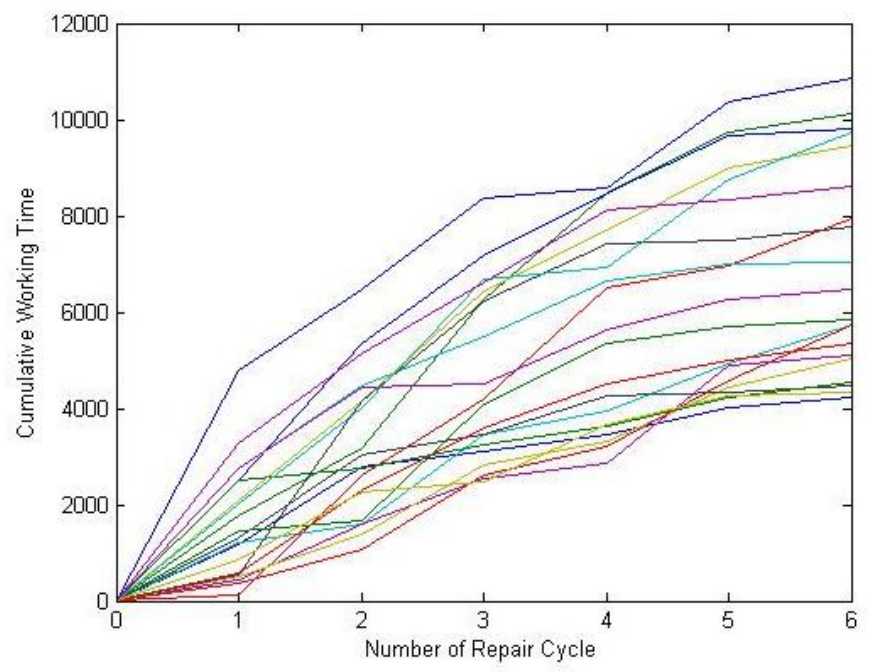

Fig. 4. Cumulative working time in each renewal cycle.

Table 2. The working time before each failure with twenty renewal cycles.

\begin{tabular}{ccccccc}
\hline Renewal & \multicolumn{6}{c}{ Norking Time During Each Failure } \\
\cline { 2 - 7 } & $X_{1}$ & $X_{2}$ & $X_{3}$ & $X_{4}$ & $X_{5}$ & $X_{6}$ \\
\hline 1 & 1164 & 1637 & 317 & 344 & 565 & 190 \\
2 & 1764 & 1427 & 3067 & 2197 & 1280 & 374 \\
3 & 131 & 2467 & 1585 & 2333 & 437 & 980 \\
4 & 1211 & 371 & 1874 & 482 & 999 & 796 \\
5 & 447 & 1137 & 947 & 345 & 2034 & 185 \\
6 & 511 & 892 & 1406 & 511 & 1127 & 603 \\
7 & 1316 & 1705 & 434 & 791 & 93 & 148. \\
8 & 2522 & 2842 & 1809 & 1302 & 1182 & 151 \\
9 & 2495 & 250 & 514 & 370 & 612 & 293 \\
10 & 585 & 1704 & 1311 & 905 & 496 & 356 \\
11 & 2768 & 1713 & 996 & 1182 & 335 & 36 \\
12 & 2753 & 1679 & 59 & 1149 & 621 & 193 \\
13 & 2079 & 2092 & 2261 & 1281 & 1283 & 441 \\
14 & 530 & 3633 & 2055 & 1197 & 89 & 255 \\
15 & 4797 & 1693 & 1872 & 201 & 1803 & 502 \\
16 & 1462 & 199 & 2408 & 1273 & 343 & 138 \\
17 & 383 & 670 & 1546 & 596 & 1367 & 1164 \\
18 & 2010 & 2023 & 2642 & 264 & 1818 & 960 \\
19 & 3264 & 1884 & 1463 & 1525 & 199 & 260 \\
20 & 850 & 1421 & 191 & 1192 & 591 & 86 \\
\hline
\end{tabular}


For a system with the same deteriorating mechanism, a sensitivity analysis is conducted to study the influence of the maintenance costs on the optimal values of the decision parameters. Three cases are considered.

1) Costly preventive maintenance: $\mathrm{A}$ high $\mathrm{PM} \operatorname{cost} C_{p}$ leads to less frequent PM operations and keeps the system working with an increased risk of failure. The influence of the PM cost on the optimal values is shown in Table 3. It can be seen that as the PM cost becomes higher and higher, the critical reliability decreases. As the critical reliability drops to very low, though larger than zero, there will be no PM in a repair cycle.

Table 3. The influence of the PM cost on the optimal values.

\begin{tabular}{cccc}
\hline$\left(C_{p}, c_{f}, \bar{c}, C\right)$ & $R^{*}$ & $N^{*}$ & $C\left(R^{*}, N^{*}\right)$ \\
\hline$(4000,100,10000,500000)$ & 0.6712 & 6 & 77.3513 \\
$(6000,100,10000,500000)$ & 0.6267 & 6 & 79.1821 \\
$(8000,100,10000,500000)$ & 0.5833 & 6 & 80.7297 \\
$(10000,100,10000,500000)$ & 0.5406 & 6 & 82.0502 \\
$(15000,100,10000,500000)$ & 0.3783 & 7 & 84.3849 \\
$(20000,100,10000,500000)$ & 0.2735 & 7 & 85.7446 \\
\hline
\end{tabular}

2) High corrective maintenance cost rate $c_{f}$ : As the $\mathrm{CM}$ cost rate increases, the $\mathrm{CM}$ operation will be more and more expensive because of, e.g., the unavailability, production losses and unplanned intervention. In this case, the optimal policy sets a high critical reliability and involves more PM operations in order to avoid system unavailability. The increasing optimal thresholds and the decreasing repair numbers are listed in Table 4. The failure damage $\bar{c}$ has a similar influence on the optimal values and, limited by the space, we will not repeat here. 
Table 4. The influence of the CM cost rate on the optimal values.

\begin{tabular}{cccc}
\hline$\left(C_{p}, c_{f}, \bar{c}, C\right)$ & $R^{*}$ & $N^{*}$ & $C\left(R^{*}, N^{*}\right)$ \\
\hline$(5000,70,10000,500000)$ & 0.5519 & 9 & 68.4320 \\
$(5000,90,10000,500000)$ & 0.6514 & 6 & 75.5480 \\
$(5000,110,10000,500000)$ & 0.6922 & 5 & 81.0063 \\
$(5000,150,10000,500000)$ & 0.6871 & 5 & 89.5926 \\
$(5000,200,10000,500000)$ & 0.7345 & 4 & 97.7157 \\
$(5000,250,10000,500000)$ & 0.7876 & 3 & 105.4958 \\
\hline
\end{tabular}

3) Costly replacement $C$ : As shown in Table 5, the PM cost has its influence mainly on the number of corrective maintenance. The more expensive the replacement is, the more sensitive the optimal repair number $N^{*}$ will be to variations of $C$.

Table 5. The influence of the PM cost on the optimal values.

\begin{tabular}{cccc}
\hline$\left(C_{p}, C_{f}, \bar{c}, C\right)$ & $R^{*}$ & $N^{*}$ & $C\left(R^{*}, N^{*}\right)$ \\
\hline$(5000,100,10000,50000)$ & 0.6319 & 2 & 24.5419 \\
$(5000,100,10000,80000)$ & 0.6887 & 2 & 30.5608 \\
$(5000,100,10000,100000)$ & 0.6393 & 3 & 34.1099 \\
$(5000,100,10000,300000)$ & 0.6922 & 4 & 59.4617 \\
$(5000,100,10000,600000)$ & 0.6314 & 7 & 86.3033 \\
$(5000,100,10000,800000)$ & 0.5877 & 10 & 98.7854 \\
\hline
\end{tabular}

\section{Concluding remarks}

In this paper a multi-state deteriorating system is studied and an optimal bivariate maintenance policy, namely $(R, N)$, is developed. This multi-state system has $k$ failure states and one working state. This is a general formulation and many systems can be described in this way with a suitable failure and state specification. Under some commonly used assumptions, we have derived an explicit expression of the average cost rate function, and thus the optimal solutions can be readily obtained. 
The maintenance strategy proposed herein is very flexible, including many maintenance policies as special cases. The PM can be assumed to be perfect, in which case we have $a=b=$ 1. If the threshold reliability is set to be zero, namely no preventive maintenance is taken, then our model reduces to the repair number counting policy. If we do not take into consider the failure types when we are making maintenance decisions, we can set $k=1$. As these maintenance policies are particular cases, it is obvious that the proposed Possible extensions of the research could be to

1) Analyze systems with multiple components and/or more than one working state;

2) The probability of occurrences of these $k$ failure states are not constant but depend on the working time;

3) Combine other imperfect maintenance treatment methods with monotone process model, such as virtual age method, improvement factor method, multiple $(p, q)$ rule and so on.

\section{Appendix A. Proof of Theorem 1}

It is obvious that

$$
P\left(V_{n}=v_{n}\right)=R^{v_{n}}(1-R), \quad v_{n}=0,1, \ldots
$$

and that

$$
P\left(X_{n} \leq t \mid V_{1}=v_{1}, \ldots, V_{n}=v_{n}, S_{1}=s_{1}, \ldots, S_{n-1}=s_{n-1}\right)=\frac{F\left(a^{\sum_{i=1}^{n} v_{i}} \prod_{i=1}^{n-1} a_{s_{i}} t\right)}{1-R}
$$

Because the numbers of preventive maintenance in each repair cycle are independent, we have 


$$
\begin{aligned}
& P\left(V_{1}=v_{1}, \ldots, V_{n}=v_{n}, S_{1}=s_{1}, \ldots, S_{n-1}=s_{n-1}\right) \\
& =\prod_{i=1}^{n} P\left(V_{i}=v_{i}\right) \prod_{j=1}^{n-1} P\left(S_{j}=s_{j}\right) \\
& =R^{\sum_{i=1}^{n} v_{i}}(1-R)^{n} \prod_{i=1}^{n-1} p_{s_{i}} .
\end{aligned}
$$

For $0<t<\tau_{n+1}$,

$$
\begin{aligned}
P\left(X_{n+1} \leq t\right) & =\sum_{v_{1}=0}^{+\infty} \ldots \sum_{v_{n+1}=0}^{+\infty} \sum_{s_{1}=1}^{k} \ldots \sum_{s_{n}=1}^{k} F\left(a^{\sum_{i=1}^{n+1} v_{i}} \prod_{i=1}^{n} a_{s_{i}} t\right) \times R^{\sum_{i=1}^{n+1} v_{i}}(1-R)^{n} \prod_{i=1}^{n} p_{s_{i}} \\
& \geq \sum_{v_{1}=0}^{+\infty} \ldots \sum_{v_{n+1}=0}^{+\infty} \sum_{s_{1}=1}^{k} \ldots \sum_{s_{n}=1}^{k} F\left(a^{\sum_{i=1}^{n} v_{i}} \prod_{i=1}^{n-1} a_{s_{i}} t\right) \times R^{\sum_{i=1}^{n+1} v_{i}}(1-R)^{n} \prod_{i=1}^{n} p_{s_{i}} \\
& =\sum_{v_{1}=0}^{+\infty} \ldots \sum_{v_{n}=0}^{+\infty} \sum_{s_{1}=1}^{k} \ldots \sum_{s_{n-1}=1}^{k} F\left(a^{\sum_{i=1}^{n} v_{i}} \prod_{i=1}^{n-1} a_{s_{i}} t\right) \times R^{\sum_{i=1}^{n} v_{i}}(1-R)^{n-1} \prod_{i=1}^{n-1} p_{s_{i}} \\
& =P\left(X_{n} \leq t\right) .
\end{aligned}
$$

For $t \geq \tau_{n+1}, P\left(X_{n+1} \leq t\right)=1 \geq P\left(X_{n} \leq t\right)$. So we can say that $P\left(X_{n}>t\right) \geq P\left(X_{n+1}>t\right)$ and that $\left\{X_{1}, X_{2}, \ldots, X_{n} \ldots\right\}$ is a monotone decreasing process.

Similarly, for $t>0$, we have 


$$
\begin{aligned}
P\left(Y_{n+1} \leq t\right)= & \sum_{v_{1}=0}^{+\infty} \ldots \sum_{v_{n+1}=0}^{+\infty} \sum_{s_{1}=1}^{k} \ldots \sum_{s_{n+1}=1}^{k} G\left(b^{\sum_{i=1}^{n+1} v_{i}} \prod_{i=1}^{n+1} b_{s_{i}} t\right) \times R^{\sum_{i=1}^{n+1} v_{i}}(1-R)^{n+1} \prod_{i=1}^{n+1} p_{s_{i}} \\
& \leq \sum_{v_{1}=0}^{+\infty} \ldots \sum_{v_{n+1}=0}^{+\infty} \sum_{s_{1}=1}^{k} \ldots \sum_{s_{n+1}=1}^{k} G\left(b^{\sum_{i=1}^{n} v_{i}} \prod_{i=1}^{n} b_{s_{i}} t\right) \times R^{\sum_{i=1}^{n+1} v_{i}}(1-R)^{n+1} \prod_{i=1}^{n+1} p_{s_{i}} \\
& =\sum_{v_{1}=0}^{+\infty} \sum_{v_{n}=0}^{+\infty} \sum_{s_{1}=1}^{k} \ldots \sum_{s_{n}=1}^{k} G\left(b^{\sum_{i=1}^{n} v_{i}} \prod_{i=1}^{n} b_{s_{i}} t\right) \times R^{\sum_{i=1}^{n} v_{i}}(1-R)^{n} \prod p_{i=1}^{n} \\
& =P\left(Y_{n} \leq t\right) .
\end{aligned}
$$

So we can say that $P\left(Y_{n}>t\right) \leq P\left(Y_{n+1}>t\right)$ and that $\left\{Y_{1}, Y_{2}, \ldots, Y_{n} \ldots\right\}$ is a monotone increasing process.

\section{Appendix B}

Expectations $E\left(\sum_{i=1}^{N} C_{i}\right)$ and $E V_{n}$ are easy to derive and are given below:

$$
\begin{aligned}
& E\left(\sum_{i=1}^{N} C_{i}\right)=\sum_{i=1}^{N} E C_{i}=N \sum_{i=1}^{k} c_{i} p_{i} \\
& E V_{n}=\sum_{k=0}^{+\infty} k P\left(V_{n}=k\right)=\sum_{k=0}^{+\infty} k R^{k}(1-R)=\frac{R}{1-R} .
\end{aligned}
$$

We use the law of conditional expectation to derive the expected working time $E\left(X_{n}\right)$. First, we have conditional distribution

$$
P\left(X_{n} \leq t \mid V_{1}=v_{1}, \ldots, V_{n}=v_{n}, S_{1}=s_{1}, \ldots, S_{n-1}=s_{n-1}\right)
$$




$$
=\frac{F\left(a^{\sum_{i=1}^{n} v_{i}} \prod_{i=1}^{n-1} a_{s_{i}} t\right)}{1-R}, \quad 0<t \leq \tau_{n}
$$

and conditional expectation

$$
\begin{aligned}
& E\left[X_{n} \mid V_{1}=v_{1}, \ldots, V_{n}=v_{n}, S_{1}=s_{1}, \ldots, S_{n-1}=s_{n-1}\right] \\
& =\frac{1}{1-R} \int_{0}^{\tau_{n}} t d F\left(a^{\sum_{i=1}^{n} v_{i}} \prod_{i=1}^{n-1} a_{s_{i}} t\right) \\
& =\frac{\int_{0}^{F^{-1}(1-R)} t d F(t) /(1-R)}{a^{\sum_{i=1}^{n} v_{i}} \prod_{i=1}^{n-1} a_{s_{i}}} \\
& =\frac{\lambda(R) /(1-R)}{a^{\sum_{i=1}^{n} v_{i}} \prod_{i=1}^{n-1} a_{s_{i}}}
\end{aligned}
$$

where $\lambda(R)=\int_{0}^{F^{-1}(1-R)} t d F(t)$.

The expected working time $E\left(X_{n}\right)$ can be written as

$$
\begin{aligned}
& E\left(X_{n}\right) \\
& =E_{V_{1}, V_{2}, \ldots, V_{n}, S_{1}, S_{2}, \ldots, S_{n-1}}\left[E\left[X_{n} \mid V_{1}, V_{2}, \ldots, V_{n}, S_{1}, S_{2}, \ldots, S_{n-1}\right]\right] \\
& =E_{V_{1}, V_{2}, \ldots, V_{n}, S_{1}, S_{2}, \ldots, S_{n-1}}\left[\frac{\lambda(R) /(1-R)}{a^{\sum_{i=1}^{n} V_{i}} \prod_{i=1}^{n-1} a_{S_{i}}}\right] \\
& =\sum_{v_{1}=0}^{+\infty} \ldots \sum_{v_{n}=0}^{+\infty} \sum_{S_{1}=1}^{k} \ldots \sum_{s_{n-1}=1}^{k} \frac{\lambda(R) /(1-R)}{a^{\sum_{i=1}^{n} v_{i}} \prod_{i=1}^{n-1} a_{S_{i}}} \times R^{\sum_{i=1}^{n} v_{i}}(1-R)^{n} \prod_{i=1}^{n-1} p_{S_{i}} \\
& =\left(\frac{p_{1}}{a_{1}}+\cdots+\frac{p_{k}}{a_{k}}\right)^{n-1}(1-R)^{n-1} \frac{\lambda(R) a^{n}}{(a-R)^{n}}, \quad a>R .
\end{aligned}
$$


The condition $a>R$ can be removed since $a>1$ and $1>R$. Similarly, we use the law of conditional expectation to derive the expected working time $E\left(x_{n}^{j}\right)$ and the expected repair time $E\left(Y_{n}\right)$

Because

$$
E\left(x_{n}^{j} \mid V_{1}=v_{1}, \ldots, V_{n}=v_{n}, S_{1}=s_{1}, \ldots, S_{n-1}=s_{n-1}\right)=\frac{F^{-1}(1-R)}{a^{\sum_{i=1}^{n-1} v_{i}+j-1} \prod_{i=1}^{n-1} a_{s_{i}}},
$$

we have

$$
\begin{aligned}
E\left(x_{n}^{j}\right)=\sum_{v_{1}=0}^{+\infty} \ldots & \sum_{v_{n-1}=0}^{+\infty} \sum_{v_{n}=j}^{+\infty} \sum_{s_{1}=1}^{k} \ldots \sum_{s_{n-1}=1}^{k} \frac{F^{-1}(1-R)}{a^{\sum_{i=1}^{n-1} v_{i}+j-1} \prod_{i=1}^{n-1} a_{s_{i}}} \times R^{\sum_{i=1}^{n} v_{i}}(1-R)^{n} \prod_{i=1}^{n-1} p_{s_{i}} \\
= & \left(\frac{p_{1}}{a_{1}}+\cdots+\frac{p_{k}}{a_{k}}\right)^{n-1}(1-R)^{n-1}\left(\frac{a}{a-R}\right)^{n-1} \frac{R^{j} \times F^{-1}(1-R)}{a^{j-1}}, \quad j=1, \ldots, v_{n} .
\end{aligned}
$$

Because

$$
\begin{aligned}
& E\left[Y_{n} \mid V_{1}=v_{1}, \ldots, V_{n}=v_{n}, S_{1}=s_{1}, \ldots, S_{n}=s_{n}\right] \\
& =\int_{0}^{+\infty} t d G\left(b^{\sum_{i=1}^{n} v_{i}} \prod_{i=1}^{n} b_{S_{i}} t\right) \\
& =\frac{\int_{0}^{+\infty} t d G(t)}{b^{\sum_{i=1}^{n} v_{i}} \prod_{i=1}^{n} b_{s_{i}}} \\
& =\frac{u}{b^{\sum_{i=1}^{n} v_{i}} \prod_{i=1}^{n} b_{s_{i}}}, \quad u=\int_{0}^{+\infty} t d G(t),
\end{aligned}
$$

so that 


$$
\begin{aligned}
& E\left(Y_{n}\right) \\
& =\sum_{v_{1}=0}^{+\infty} \ldots \sum_{v_{n}=0}^{+\infty} \sum_{s_{1}=1}^{k} \ldots \sum_{s_{n}=1}^{k} \frac{u}{b^{\sum_{i=1}^{n} v_{i}} \prod_{i=1}^{n} b_{s_{i}}} \times R^{\sum_{i=1}^{n} v_{i}}(1-R)^{n} \prod_{i=1}^{n} p_{s_{i}} \\
& =(1-R)^{n} \frac{u b^{n}}{(b-R)^{n}}\left(\frac{p_{1}}{b_{1}}+\cdots+\frac{p_{k}}{b_{k}}\right)^{n}, \quad b>R .
\end{aligned}
$$

The condition $b>R$ is in line with reality. Since PM is always effective, restoring a system to a very good state. And due to the increasing power of $b^{\sum_{i=1}^{n} v_{i}} \prod_{i=1}^{n} b_{s_{i}}$, the value of $b$ should be close to one.

\section{Appendix C. Proof of Theorem 2}

$$
\begin{aligned}
& \sum_{i=1}^{N} E X_{i}=\sum_{i=1}^{N} A^{i-1}(1-R)^{i-1} \frac{\lambda(R) a^{i}}{(a-R)^{i}}=\frac{\lambda(R) a}{a-R} \times \frac{1-\left[A(1-R) \frac{a}{a-R}\right]^{N}}{1-A(1-R) \frac{a}{a-R}} ; \\
& \sum_{i=1}^{N-1} E Y_{i}=\sum_{i=1}^{N-1} B^{i}(1-R)^{i} \frac{u b^{i}}{(b-R)^{i}}=B(1-R) \frac{u b}{b-R} \times \frac{1-\left[B(1-R) \frac{b}{b-R}\right]^{N-1}}{1-B(1-R) \frac{b}{b-R}} ;
\end{aligned}
$$




$$
\begin{aligned}
& \sum_{n=1}^{N} E\left(\sum_{j=1}^{V_{n}} x_{n}^{j}\right) \\
& =\sum_{n=1}^{N}\left[\sum_{v_{1}=0}^{+\infty} \ldots \sum_{v_{n-1}=0}^{+\infty} \sum_{v_{n}=1}^{+\infty} \sum_{s_{1}=1}^{k} \ldots \sum_{s_{n-1}=1}^{k}\left(\sum_{j=1}^{v_{n}} \frac{F^{-1}(1-R)}{a^{\sum_{i=1}^{n-1} v_{i}+j-1} \prod_{i=1}^{n-1} a_{s_{i}}}\right) \times R^{\sum_{i=1}^{n} v_{i}}(1-R)^{n} \prod_{i=1}^{n-1} p_{s_{i}}\right] \\
& =\sum_{n=1}^{N}\left[\sum_{v_{1}=0}^{+\infty} \ldots \sum_{v_{n-1}=0}^{+\infty} \sum_{v_{n}=1}^{+\infty} \sum_{s_{1}=1}^{k} \ldots \sum_{s_{n-1}=1}^{k}\left(\frac{F^{-1}(1-R)}{a^{\sum_{i=1}^{n-1} v_{i}} \prod_{i=1}^{n-1} a_{s_{i}}} \times \frac{1-(1 / a)^{v_{n}}}{1-1 / a}\right) \times R^{\sum_{i=1}^{n} v_{i}}(1-R)^{n} \prod_{i=1}^{n-1} p_{s_{i}}\right] \\
& =\sum_{n=1}^{N} \frac{F^{-1}(1-R)}{a-1} \times\left(a-\frac{a^{2}(1-R)}{a-R}\right) \times A^{n-1} \times\left(\frac{a}{a-R}\right)^{n-1} \times(1-R)^{n-1} \\
& =\frac{F^{-1}(1-R) \times a R}{a-R} \times \frac{1-\left[A(1-R) \frac{a}{a-R}\right]^{N}}{1-A(1-R) \frac{a}{a-R}} .
\end{aligned}
$$

Acknowledgement: The work described in this paper was mainly supported by a grant from City University of Hong Kong (Project No.9380058) and a grant from the Research Grants

Council of the Hong Kong Special Administrative Region, China (CityU8/CRF/09). Prof Gaudoin's work was supported by the French National Agency of Research (ANR), project AMMSI, number ANR-2011-BS01-021.

\section{References}

[1] N. Gebraeel, A. Elwany and J. Pan, "Residual life predictions in the absence of prior degradation knowledge,” IEEE Transactions on Reliability, vol. 58, pp. 106-117, 2009. 
[2] P.E. Labeau and M.C. Segovia, "Effective age models for imperfect maintenance," Proceedings of the Institution of Mechanical Engineers, Part O-Journal of Risk and Reliability, vol. 225, pp. 117-130, 2011.

[3] F.Q. Yuan and U. Kumar, "A general imperfect repair model considering time-dependent repair effectiveness," IEEE Transactions on Reliability, vol. 61, pp. 95-100, 2012.

[4] Y. Liu, H.Z. Huang and X.L. Zhang, "A data-driven approach to selecting imperfect maintenance models,” IEEE Transactions on Reliability, vol. 61, pp. 101-112, 2012.

[5] Y. Lam, "A note on the optimal replacement problem," Advances in Applied Probability, vol. 20, pp. 479-482, 1988.

[6] H.Z. Wang and H. Pham, "A quasi renewal process and its applications in imperfect maintenance,” International Journal of Systems Science, vol. 27, pp. 1055-1062, 1996.

[7] H.Z. Wang and H. Pham, "Reliability and optimal maintenance," London: Springer, 2006.

[8] Y. Lam, "A geometric process delta-shock maintenance model," IEEE Transactions on Reliability, vol. 58, pp. 389-396, 2009.

[9] J.S. Jia and S.M. Wu, "A replacement policy for a repairable system with its repairman having multiple vacations," Computers and Industrial Engineering, vol. 57, pp. 156-160, 2009a.

[10]J.S. Jia and S.M. Wu, "Optimizing replacement policy for a cold-standby system with waiting repair times," Applied Mathematics and Computation, vol. 214, pp. 133-141, $2009 \mathrm{~b}$. 
[11]Y.P. Wang and H. Pham, "A multi-objective optimization of imperfect preventive maintenance policy for dependent competing risk systems with hidden failure," IEEE Transactions on Reliability, vol. 60, pp. 770-781, 2011.

[12]L. Doyen and O. Gaudoin, "Modeling and assessment of aging and efficiency of corrective and planned preventive maintenance," IEEE Transactions on Reliability, vol. 60, pp. 759-769, 2011.

[13]C. Derman, "On optimal replacement rules when changes of state are Markovian," Mathematical Optimization Techniques, R. Bellman (ed.), The RAND Corporation, 1963.

[14]J. Wu, Ng T.S. Adam, M. Xie and H.Z. Huang, "Analysis of maintenance policies for finite life-cycle multi-state systems," Computers and Industrial Engineering, vol. 59, pp. 638-646, 2010.

[15]Y. Liu and H.Z. Huang, "Optimal replacement policy for multi-state system under imperfect maintenance," IEEE Transactions on Reliability, vol. 59, pp. 483-495, 2010.

[16]M. Xu, T. Chen and X.H. Yang, "Optimal replacement policy for safety-related multicomponent multi-state systems," Reliability Engineering and System Safety, vol. 99, pp. 87-95, 2012.

[17]R. Moghaddass, M.J. Zuo and M. Pandey, "Optimal design and maintenance of a repairable multi-state system with standby components," Journal of Statistical Planning and Inference, vol. 142, pp. 2409-2420, 2012. 
[18]Y.L. Zhang, and G.J. Wang, “An extended replacement policy for a deteriorating system with multi-failure modes," Applied Mathematics and Computation, vol. 218, pp. 18201830, 2011.

[19]Y. Lam, Y.L. Zhang and Y.H. Zheng, "A geometric process equivalent model for a multistate degenerative system," European Journal of Operational Research, vol. 142, pp. 21-29, 2002.

[20]Y.L. Zhang, R.C.M. Yam and M.J. Zuo, “A bivariate optimal replacement policy for a multistate repairable system," Reliability Engineering and System Safety, vol. 92, pp. 535-542, 2007.

[21]S. Sheu, W.S. Griffith and T. Nakagawa, "Extended optimal replacement model with random minimal repair costs," European Journal of Operational Research, vol. 85, pp. 636-49, 1995.

[22]H.Z. Wang, "A survey of maintenance policies of deteriorating systems," European Journal of Operational Research, vol. 139, pp. 469-489, 2002.

[23]X.J. Zhou, L.F. Xi and J. Lee, "Reliability-centered predictive maintenance scheduling for a continuously monitored system subject to degradation," Reliability Engineering and System Safety, vol. 92, pp. 530-534, 2007.

[24]W.Z. Liao, E. Pan and L.F. Xi, "Preventive maintenance scheduling for repairable system with deterioration," Journal of Intelligent Manufacturing, vol. 21, pp. 875-884, 2010.

[25]S.M. Ross, “Stochastic processes,” Wiley, New York, 1983. 
[26]S.M. Ross, "Introduction to probability models," Academic Press, 2007. 